\title{
Classical light analogue of the nonlocal Aharonov-Bohm effect
}

\author{
Nandan Satapathy ${ }^{1}$, Deepak Pandey ${ }^{1}$, Poonam Mehta $^{1,2}$, Supurna Sinha $^{1}$, \\ Joseph Samuel ${ }^{1}$ and Hema Ramachandran ${ }^{1}$ \\ 1 Raman Research Institute, C. V. Raman Avenue, Sadashivanagar, Bangalore, India 560080. \\ 2 Presently at the Department of Physics and Astrophysics, University of Delhi, Delhi, India $11000 \%$.
}

\begin{abstract}
PACS 03.65.Vf - Phases: geometric; dynamic or topological
PACS 25.75.Gz - Particle correlations and fluctuations

PACS 95.75. Kk - Interferometry

Abstract - We demonstrate the existence of a non-local geometric phase in the intensity-intensity correlations of classical incoherent light, that is not seen in the lower order correlations. This twophoton Pancharatnam phase was observed and modulated in a Mach-Zehnder interferometer. Using acousto-optic interaction, independent phase noise was introduced to light in the two arms of the interferometer to create two independent incoherent classical sources from laser light. The experiment is the classical optical analogue of the multi-particle Aharonov-Bohm effect. As the trajectory of light over the Poincaré sphere introduces a phase shift observable only in the intensityintensity correlation, it provides a means of deflecting the two-photon wavefront, while having no effect on single photons.
\end{abstract}

Introduction: Two classic interference experiments are Young's double-slit experiment and the Hanbury Brown and Twiss (HB-T) experiment. The former measures the amplitude-amplitude correlation and demonstrates the interference of a photon with itself. The latter experiment measures the intensity-intensity correlation and is a $\checkmark$ demonstration of the interference of a pair of photons with jitself. While the HB-T intensity-interferometry experiment was historically important in leading to our present understanding of quantum optics and coherence, one does - not need to invoke quantum mechanics to understand it; it can quite simply be understood entirely in terms of classical electric field fluctuations. In recent times, with the development of different types of light sources and detection techniques, intensity-interferometry has, once again, become a topic of great interest. It has led to many tantalizing ideas and interesting applications 1 -5]. There has been enormous debate 6 [ 9 on the similarities and differences between bi-photon interferometry and two-photon inerferometery. Bi-photon interferometry $10-12$ uses entangled photon pairs, i.e., pairs of photons that are related due to a conservation principle, for example, product photons in parametric down-conversion. Two-photon interferometry 13, on the other hand, involves photons that are statistically correlated, as from a thermal source; our present experiment belongs to this category. Intensityinterferometry with classical light is regaining importance with the demonstration of ghost imaging with thermal light 14 18].

A classical HB-T experiment consists of two slits, each illuminated by an independent, incoherent light source. Two detectors measure the intensity of light falling on them; at each detector, the intensity has contributions from both slits. The cross correlation of the intensities at the two detectors varies in a sinusoidal fashion as a function of separation between the detectors. In the conventional HB-T experiment, the appearance of these fringes was a purely dynamical effect, arising from the change in the path difference of the two detectors from the two slits. Recently it was shown theoretically [19 that even with fixed detectors, one can have such fringes if the sources and detectors are polarised and the polarisation is varied. This is purely a geometric or Pancharatnam phase effect 20,21 and arises due to the closed trajectory of polarised light on the Poincaré sphere. While such geometric phase effects are well known in amplitude interferometry, their counterparts in intensity interferometry have been less studied. The geometric phase is given by half the solid angle subtended by a closed path traced out by polarising elements on the Poincaré sphere (see Fig. 11). Unlike the dynamical phase shift that is restricted by the spatial and temporal coherence of the sources, the geometric phase, is achromatic and unbounded, as will be discussed shortly.

In this Letter, we wish to convey several points. We 


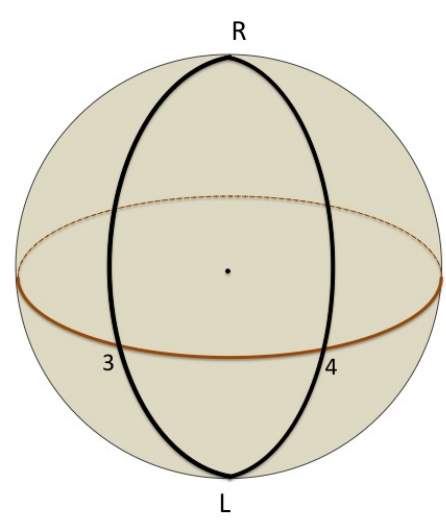

Fig. 1: (Colour online) The Poincaré sphere. Figure shows four points on the Poincare sphere representing two circular polarisation states $(R, L)$ and two linear polarisation states of the two polarisers in front of the two detectors $(3,4)$. The geometric phase is given by half the solid angle subtended at the centre, by the geodesic polygon R-4-L-3-R.

demonstrate a new way of producing classical light with exactly tailored characteristics. This is achieved by creating radio-frequency electrical signals of the desired features and imprinting this onto light via acousto-optic interaction. We use this technique to create light that has random phase noise only. Next, we show that a small modification to the HB-T intensity interferometry experiment leads to an optical analogue of the multiparticle Aharonov-Bohm (AB) effect 22]. This manifests as a non-local geometric phase in the intensity-intensity correlations of classical incoherent light, that is not seen in the lower order correlations. Finally, we show that this non-local two-photon cross-correlation can be modulated via the geometric phase. This suggests a way of deflecting the cross-correlated photon pairs.

The AB effect 23,24 shows that the electromagnetic potential potential affects charged particles even in regions where no field exists. It is seen as a phase acquired by the wave function of an electron when it moves in a path enclosing a solenoid. The path is entirely in a region where the magnetic field is zero and the acquired phase is attributed to the enclosed flux 25]. The AB effect led to a wide appreciation of the role of the electromagnetic potential, in particular its interpretation [26] as a "connection", a structure well studied by mathematicians in differential geometry. A connection is a rule for comparing quantities defined at different points 1 . The $\mathrm{AB}$ effect can be viewed as a physical manifestation of a flat connection, since there is no magnetic field in the region accessible to the electron. The AB effect has both geometric and topological aspects to it. It is insensitive to small deformations of the interfering paths and therefore may be viewed as topological.

\footnotetext{
${ }^{1} \mathrm{~A}$ connection need not be integrable along a closed curve and this lack of integrability is called a holonomy. If the connection is locally integrable it has no curvature and is said to be flat.
}

At the same time, it is sensitive to small changes of the magnetic flux and in this sense is geometric.

Essentially the same mathematical structure underlies the Pancharatnam phase, seen, for example [27], in a Sagnac interferometer, where the dynamic path difference is zero. This geometric phase is a consequence of the solid angle enclosed on the Poincaré sphere. It too is insensitive to small changes in the paths of the interfering beams and hence is topological, while being sensitive to small changes in the solid angle enclosed on the Poincaré sphere is also geometric. There is thus a close analogy between the Pancharatnam phase and the $\mathrm{AB}$ phase.

While the Pancharatnam phase predates the AB phase, the latter has recently been extended to non local multiparticle effects which intriguingly, are manifest only in the cross correlations, and not in the self correlations or in the lower order correlations. Considered theoretically for a pair of electrons by Samuelsson et al 22, this twoparticle non-local AB effect was observed experimentally by Neder et al 28]. A pair of electrons together enclosed an $\mathrm{AB}$ flux due to an applied magnetic field, which was then used to modulate the two particle cross correlation via coupling to the orbital degree of freedom of electrons while the spin remained frozen. The effects of the flux were absent in the individual currents and in their self correlations while the cross correlation between electron currents revealed the dependence on the AB flux.

The experiment of Neder et al was essentially quantum in nature, as electrons, being fermions, do not admit the limit of large particle numbers. In contrast to fermions, bosons permit macroscopic occupancy of a single state, admitting a classical limit and permitting a classical field theory description. The experiment that we report here is the classical bosonic analogue of the two-particle nonlocal AB effect. Classical light beams from two sources meet after having traversed two independent paths over the Poincaré sphere and together enclose a solid angle on the sphere. One of the polarising elements was varied so as to modulate the enclosed solid angle, and the first order correlation (interference visibility), the second order selfcorrelation and the second order cross-correlations were measured. The effect of the modulation of the geometric phase was seen only in the second order cross correlation and not in the other measured quantities. Thus, apart from the sign differences owing to the different statistics of the particles involved, the effect that we report here and the two-particle non-local AB effect are conceptually very similar.

Experimental Setup: Our experiment employs two phase-incoherent sources $S 1$ and $S 2$ in a Mach-Zehnder setup with birefringent elements in the two arms, and polarisers preceding two detectors D3 and D4 (see Fig. 2). A variation of the relative polarisation angle of the detectors introduces a geometric phase equal to half the solid angle enclosed by the two interfering paths on the Poincaré sphere (see Fig. 1). Light from a diode laser at $767 \mathrm{~nm}$ was incident on a non-polarising beam- 


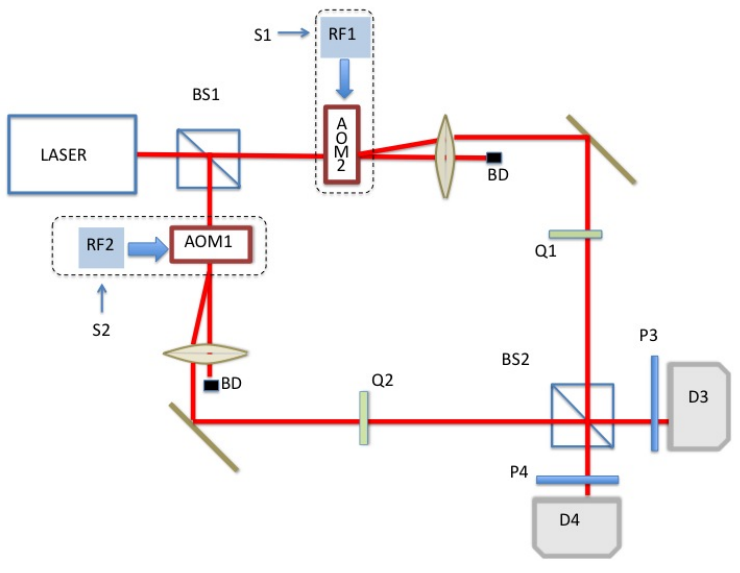

Fig. 2: (Colour online) Figure shows a schematic diagram of the experimental setup. Two sources $S 1$ and $S 2$ illuminate two detectors $D 3$ and $D 4$ as explained in the text. $B S 1$ and $B S 2$ are non-polarising beam splitters, $Q 1$ and $Q 2$ are quarter wave plates, $P 3$ and $P 4$ are linear polarisers and $B D$ are beam dumps.

splitter depicted as BS1 in Fig. 2, and the two emergent beams were passed through two acousto-optic-modulators (AOM) shown in Fig. 2 as AOM1 and AOM2, fed by two radio frequency sources (RF1 and RF2) respectively. The first order diffracted beams emerging from the two AOMs were passed through two quarter-wave plates $(Q 1, Q 2)$, to render one beam right circularly polarised $(\mathrm{R})$ and the other left-circularly polarised (L). The two beams were then combined at a non-polarising beam splitter (BS2). At each exit face of the final beam splitter, a polariser $(P 3, P 4)$ was kept, followed by a detector $(D 3, D 4)$. The undiffracted beams terminated in beam dumps (BD) as shown in Fig. 2. By keeping one of the polarisers (e.g., P3 ) fixed and changing the orientation of the other polariser $(P 4)$, the relative angle between the polarisers, $\phi_{34}$ could be varied continuously from $0^{\circ}$ to $360^{\circ}$. This results in a corresponding variation in the geometric phase. As we show below, this also causes a modulation of the intensity cross-correlation of the light reaching the two detectors. In order to measure this, the intensities of light reaching detectors $D 3$ and $D 4$, for each orientation of $P 4$, was recorded for a certain length of time on a digital storage oscilloscope and cross correlated offline. Thus, from the recorded time series, one may determine first and second order correlations.

In striking contrast to the other interference experiments which require a source of coherent light, the twophoton intensity-interferometery needs incoherent sources as HB-T correlations vanish for a laser light source. One could use a thermal source such as a mercury vapour lamp. However, the coherence time of natural thermal light sources is too short for bunching effects to be discernible by present day solid-state photo-detectors. Most two-photon interferometry experiments simulate thermal light by passing laser light through a rotating ground-glass plate. This introduces, random intensity and phase fluctuations that occur at a time scale detectable by present day solid-state detectors. We employed a different technique that utilises acousto-optic interaction.

In an $\mathrm{AOM}$, a radio-frequency ( $\mathrm{RF}$ ) electrical signal is applied to create a travelling acoustic-wave grating that can diffract light. The frequency, intensity, and phase of light can be manipulated through acousto-optic interaction by suitably tailoring the RF input to the AOM. In this experiment, however, only phase fluctuations were introduced. As demonstrated recently [29], an incoherent source may be created by electronically introducing random phase jumps to the RF and imprinting these onto laser light. The phase evolves undisturbed for a time $T$, and then is changed by a random amount $\delta$. The phase jump $\delta$ is uniformly randomly distributed over the circle and $T$ has the distribution of a truncated exponential:

$$
P(T)=\frac{1}{T_{c}} e^{-\frac{T}{T_{c}}}
$$

where $1 \mu s<T<100 \mu s$, and $T_{c}=10 \mu s . T_{c}$ represents the timescale over which the coherence of the light beam is lost. Such a distribution (in untruncated form) has been discussed [30] in connection with the emission of a single atom interrupted by collisions. Each AOM in the setup was independently phase modulated in this manner to derive two independent phase-incoherent sources (S1 and S2 in Fig. 22 from the same laser light.

Theory: We now examine $E_{3}$ and $E_{4}$, the light fields reaching detectors D3 and D4, in terms of $E_{1}$ and $E_{2}$, the light fields emerging from Q1 and Q2. Using the helicity basis vectors $|R\rangle=\left(\begin{array}{l}1 \\ 0\end{array}\right)$ and $|L\rangle=\left(\begin{array}{l}0 \\ 1\end{array}\right)$ we may represent linear states by $\left|\varphi_{i}\right\rangle=\frac{1}{\sqrt{2}}\left(e^{-i \varphi_{i}}|R\rangle+e^{i \varphi_{i}}|L\rangle\right)$. We introduce projection matrices $P_{K}=|K\rangle\langle K|$ onto polarisation states $|K\rangle$, where $\mathrm{K}=\mathrm{R}, \mathrm{L}, 3,4$. More explicitly,

$$
P_{R}=\left(\begin{array}{cc}
1 & 0 \\
0 & 0
\end{array}\right) ; P_{L}=\left(\begin{array}{cc}
0 & 0 \\
0 & 1
\end{array}\right) ; P_{i}=\frac{1}{2}\left(\begin{array}{cc}
1 & e^{-i 2 \varphi_{i}} \\
e^{i 2 \varphi_{i}} & 1
\end{array}\right)
$$

Representing polarisation vectors with Greek incides and using the Einstein summation convention (for the Greek, but not for the Latin indices), we can write

$$
\begin{aligned}
E_{i}^{\alpha} & =\frac{1}{\sqrt{2}} P_{i}^{\alpha \beta}\left[\epsilon_{i} P_{L}^{\beta \gamma} E_{2}^{\gamma} u_{i 2}+P_{R}^{\beta \gamma} E_{1}^{\gamma} u_{i 1}\right] \\
\bar{E}_{i}^{\alpha} & =\frac{1}{\sqrt{2}}\left[\bar{u}_{i 2} \bar{E}_{2}^{\gamma} P_{L}^{\gamma \beta} \epsilon_{i}+\bar{u}_{i 1} \bar{E}_{1}^{\gamma} P_{R}^{\gamma \beta}\right] P_{i}^{\beta \alpha}
\end{aligned}
$$

where the overbar stands for complex conjugation; $\epsilon_{i}$ is a pure phase $\epsilon_{3}=1, \epsilon_{4}=-1$ due to the geometry of the Mach-Zehnder setup. The quantities of interest for the $\mathrm{AB}$ effect are the different correlations at detectors D3 and D4. The first order correlations or intensities are

$$
\mathcal{I}_{i}^{1}(\tau=0)=\left\langle I_{i}\right\rangle=\left\langle\bar{E}_{i}^{\alpha} E_{i}^{\alpha}\right\rangle ; \quad i=3,4
$$


Note that each detector $i$ has contributions from both $E_{1}$ and $E_{2}$. In our experiment, the light intensities were adjusted so that $\left\langle\bar{E}_{1} E_{1}\right\rangle=\left\langle\bar{E}_{2} E_{2}\right\rangle$, i.e., $\left\langle I_{1}\right\rangle=\left\langle I_{2}\right\rangle$. The second order cross correlations are

$$
\mathcal{G}_{34}^{2}(\tau)=\frac{\left\langle I_{3}(0) I_{4}(\tau)\right\rangle}{\left\langle I_{3}\right\rangle\left\langle I_{4}\right\rangle}=\frac{\left\langle\bar{E}_{3}^{\alpha}(0) E_{3}^{\alpha}(0) \bar{E}_{4}^{\beta}(\tau) E_{4}^{\beta}(\tau)\right\rangle}{\left\langle\bar{E}_{3}^{\alpha} E_{3}^{\alpha}\right\rangle\left\langle\bar{E}_{4}^{\beta} E_{4}^{\beta}\right\rangle}
$$

and self correlations

$$
\mathcal{G}_{i i}^{2}(\tau)=\frac{\left\langle I_{i}(0) I_{i}(\tau)\right\rangle}{\left\langle I_{i}\right\rangle^{2}}=\frac{\left\langle\bar{E}_{i}^{\alpha}(0) E_{i}^{\alpha}(0) \bar{E}_{i}^{\beta}(\tau) E_{i}^{\beta}(\tau)\right\rangle}{\left\langle\bar{E}_{i}^{\alpha} E_{i}^{\alpha}\right\rangle^{2}}
$$

where $I_{3}(0)$ and $I_{4}(\tau)$ are the intensities measured at $D 3$ at time 0 and at $D 4$ at time $\tau$ respectively and the average $\langle\ldots\rangle$ is a time average over the integration time $T_{i n t}$, for a given setting of the polarisers $\mathrm{P} 3$ and $\mathrm{P} 4$ :

$$
\langle f\rangle=\frac{1}{T_{\text {int }}} \int_{0}^{T_{i n t}} f(t) d t
$$

Substituting in Eqs. 5 and Eq. 6 from Eq. 3 and multiplying the terms explicitly yields 16 terms, of which 10 vanish on time averaging. Use is made of the fact that the $2 \times 2$ Hermitean projection matrices $P$ satisfy $P^{2}=P$. Two of the surviving terms have a sequence of projections of the form $P_{R} P_{3} P_{L} P_{4} P_{R}$, which define a closed trajectory on the Poincaré sphere. This gives rise to the geometric phase $\Phi_{G}=\Omega / 2$, that is, half the solid angle enclosed. Simple algebra yields, for the cross correlation

$$
\mathcal{G}_{34}^{2}(\tau=0)=1-\frac{1}{2} \cos \left(\phi_{D}+\frac{\Omega}{2}\right)
$$

and for the self correlation

$$
\mathcal{G}_{i i}^{2}(\tau=0)=1+\frac{1}{2} \cos \left(\phi_{D}\right)
$$

where $\phi_{D}$ represents the dynamical phase (which, in our Mach-Zehnder interferometer has been adjusted to zero) and $\frac{\Omega}{2}$ is the geometric phase. The negative sign for the second term (in Eq. 8) is due to the Mach-Zehnder configuration.

On similar lines, using Eq. 3 in Eq. 4, the first order correlation may be evaluated. Of the four terms in the product, only two survive, with products of the form $P_{i} P_{R} P_{i},(i=3,4)$, which depend only on the intensities of the sources and not the geometric phase. Thus,

$$
\mathcal{I}_{i}^{1}=\frac{\left\langle I_{1}+I_{2}\right\rangle}{4}, \quad i=3,4
$$

The geometric phase appears only in the second order intensity cross correlation (Eq. 8), and is absent both in the second order intensity self correlation (Eq. 9p and the first order correlation (Eq. 10). Thus, this effect is analogous to the two-particle non-local AB effect.

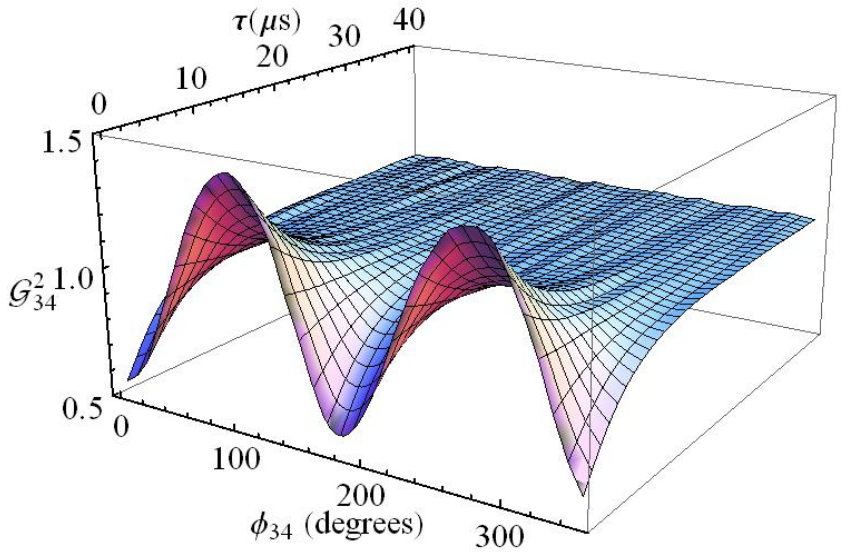

Fig. 3: (Colour online) Figure shows the experimentally determined cross correlation, $\mathcal{G}_{34}^{2}$, as a function of $\phi_{34}$ the relative angle between the polarisers $P 3$ and $P 4$, and as function of time delay $\tau$. Clearly, the cross correlation $\mathcal{G}_{34}^{2}$ is modulated by the geometric phase.

Experimental Results : The main results of our experiment are displayed in the three-dimensional plot of Fig. 3. which shows the quantity of interest, $\mathcal{G}_{34}^{2}$, as a function of the time delay, $\tau$, and the relative angle, $\phi_{34}$, between the linear polarisers $P 3$ and $P 4$ in front of the two detectors $D 3$ and $D 4$ respectively. In the experiment $P 3$ was kept fixed and $P 4$ was rotated. In effect, $\phi_{34}$ is a measure of the geometric phase.

For zero delay $(\tau=0)$ between the two detectors, the cross correlation $\mathcal{G}_{34}^{2}$ was found to vary sinusoidally from $\sim 0.5$ to $\sim 1.5$ as $\phi_{34}$ is varied. Clearly, this is due to the geometric phase $\Phi_{G}=\Omega / 2=2 \phi_{34}$. For larger values of $\tau$ the amplitude of the sinusoidal variation is found to be progressively diminished, till finally, for $\tau>T_{C}, \mathcal{G}_{34}^{2}$ remains nearly constant at 1 , i.e., correlations that are maximum for zero delay gradually decrease, and are absent for delays larger than the coherence time. At the regions of constructive intereference (e.g. $\phi_{34}=90^{\circ}$ ), the value of $\mathcal{G}_{34}^{2}$ starts from 1.5 for $\tau=0$ as is expected for a source with pure phase fluctuations 31]. Note that in regions of destructive interference, the cross correlation starts from 0.5 , a value lower than that for a coherent source. This has been discussed in various contexts earlier 32 .

In order to illustrate that it is a purely nonlocal effect we also plot in Fig. 4, the second order self correlation. In contrast to the cross-correlation, the self-correlation remains unaltered as the relative angle between the polarisers is varied. This may be easily understood by visualising the trajectory on the Poincaré sphere. For example, for $\mathcal{G}_{44}^{2}$ the trajectory is obtained by the sequence $P_{R} P_{4} P_{L} P_{4} P_{R}$ and defines an arc connecting the three points $R, 4, L$ and does not enclose any solid angle; hence the absence of a geometric phase 20.

Having established the non-local nature of this effect , we next verify its absence in the lower order correlation. Using the same experimental data that was used above, 


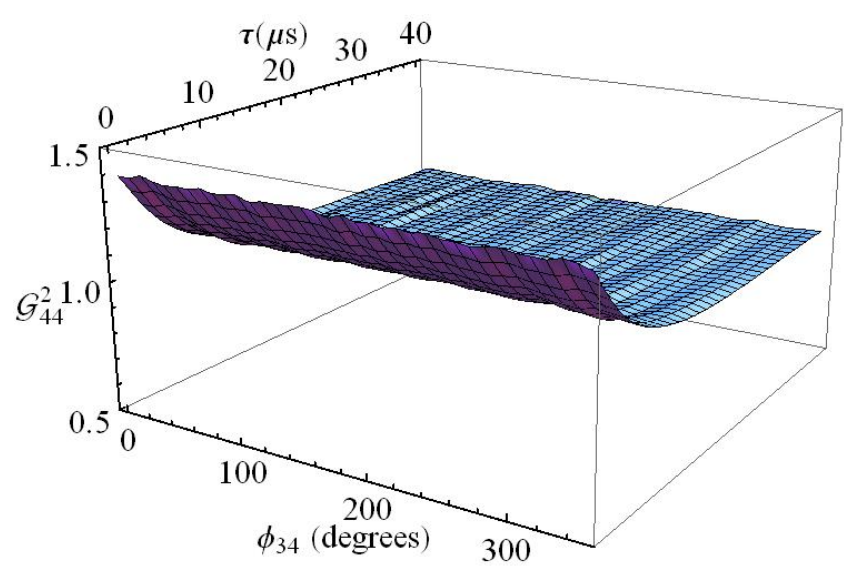

Fig. 4: (Colour online) Figure shows the self correlation $\mathcal{G}_{44}^{2}$ as a function of $\phi_{34}$ the relative angle between the polarisation angles of the two detectors, and of the time delay $\tau$. Note that the self correlation $\left(\mathcal{G}_{44}^{2}\right.$ is plotted) is independent of $\phi_{34} \cdot \phi_{34}$ is varied by turning the polaroid $\mathrm{P} 4$ in the observed channel. Thus the geometric phase effect of Fig. 3 is a purely nonlocal effect.

we now examine the quantity $\mathcal{I}_{4}^{1}$. In Fig. 5 we display the quantity $\mathcal{I}_{i}^{1}(\tau=0)$ as a function of the setting $\phi_{4}$ of the polaroid P4 in front of detector D4. Note that this curve is practically constant, showing that turning the polaroid $\mathrm{P} 4$ does not affect the lower order correlation function, that is, the geometric phase is not seen in the lower order correlations.

Discussion: The experiment and the theoretical analysis presented here are purely classical, in contrast to the earlier quantum mechanical treatment 19$]$ that predicted similar results, and the recent photon-counting intensity interferometry experiments 33 that verified it. In this Letter we develop the analogy between classical polarised light and the quantum nonlocal AB effect. Further, we explicitly show its non-local nature, and its absence in lower order correlations.

The classical approach has the advantage that the physical ideas are easy to grasp. In fact, Hanbury Brown and Twiss were originally motivated by their classical experience with radio waves to propose the corresponding optical experiment. The quantum interpretation of this classically simple experiment led to profound changes in our understanding of quantum optics and coherence.

In addition to being completely in the classical domain, our experiment has another novelty - the use of a source that has phase fluctuations only. In the case of thermal or pseudothermal light (i.e., one with both phase and intensity fluctuations), $\mathcal{G}^{2}$ varies between 1 and 2 . In contrast, for pure phase modulation, one expects a variation from 0.5 to 1.5 . Baym, in his review article 31 that examines the HB-T effect in a wide variety of physical contexts ranging from nuclear physics to astronomy, had reached this conclusion in his discussion of the case of pure phase fluctuations. However, this has hitherto not been verified, as

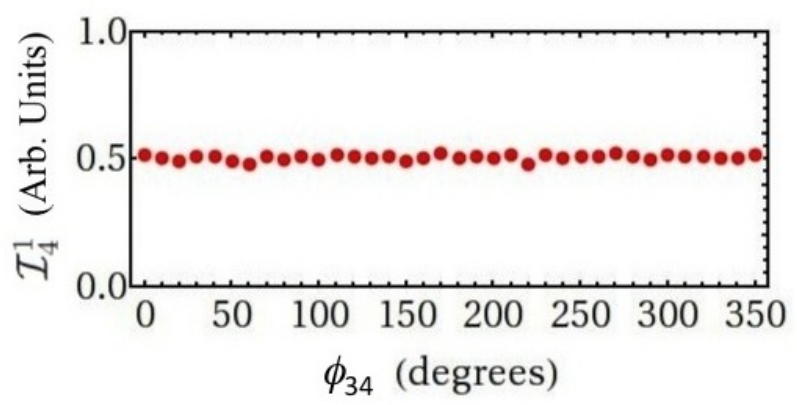

Fig. 5: (Colour online) $\mathcal{I}_{4}^{1}$ as a function of $\phi_{34}$ as obtained from the experiment. $\phi_{34}=\phi_{3}-\phi_{4}$ is varied by turning the polaroid P4 in the observed channel while keeping P3 fixed.

pure "phase-only" fluctuating sources have not been available. In our experiment, such a source has been realised by tailoring fluctuations in light by the acousto-optic technique, and the result of Baym verified.

Two salient features of the geometric phase measured in our experiment are its purely non-local nature and its absence in lower order correlations, in contrast to earlier work on geometric phase and intensity interferometry 34 . These specific features give rise to the possibility of the application of this nonlocal AB effect as a two-photon deflector. We refer again to Fig. 2, where we now replace the polariser $P 4$ by a "graded polariser" - one where the axis of polarisation changes in orientation gradually as one moves across the polariser. In intensity interferometry this would introduce a geometric phase gradient. As is well known, a phase gradient in a wave is equivalent to a deflection or a change of wave vector. In the present experiment, the phase change appears only in the intensity cross correlations. Thus, we may generate a "correlated-photon deflector" which affects the intensity-intensity correlation, leaving individual photons unaffected. Such a device may have applications in creating and manipulating correlated photons.

We close with a few classical remarks which may have interesting quantum interpretations. Let us note that the geometric phase is unbounded. As one turns the polariser $P 4$, the geometric phase keeps accumulating without bound and the visibility of the interference pattern remains undiminished. This is in sharp contrast to the dynamical phase: as one increases the dynamical phase, by separating the detectors, the interference pattern is washed out when their spatial (temporal) separation exceeds the coherence length (time). This difference is due to the fact that the geometric phase is achromatic and affects all frequencies equally. Thus the geometric phase effects are less susceptible to decoherence than the corresponding dynamical effects. This illustrates a point often made in the quantum computation literature that geometric and topological effects are robust against decoherence. This is 
crucial to the development of quantum computers.

At the level of classical information theory, our experiment can be interpreted as a way of delocalising information. Consider the experimental setup above and make the following minor changes: We choose $P 3$ and $P 4$ to be orthogonal linear polarisations represented by antipodal points on the equator of the Poincaré sphere. We take the sources to be elliptically polarised and lying on the great circle orthogonal to the line joining the antipodal points 3 and 4 . If the angle $\phi$ between the elliptical polarisations of the two sources is varied in time $\phi(t)$, one would find that there is a corresponding modulation in the crosscorrelations of the intensities detected at D3 and $D 4$. The signal $\phi(t)$ can be viewed as carrying information. However, the two beams emerging from the experiment are of fixed linear polarisation, and each beam by itself appears thermal in its self correlation. It is only by looking at their cross correlations that one can recover the originally impressed signal $\phi(t)$. Thus, in this example, information is stored in a completely delocalised manner. In quantum information theory, one expects that it would be similarly possible to have apparently thermal beams carrying information entirely in their quantum cross correlations.

To summarise, we have demonstrated the existence of a non-local geometric phase in the intensity-intensity correlations of classical incoherent light, that is not seen in the lower order correlations. This two-photon Pancharatnam phase is the classical optical analogue of the multiparticle AB effect 28. As the trajectory of light over the Poincaré sphere introduces a phase shift observable only in the intensity-intensity correlation, it provides a means of deflecting the two-photon wavefront, while having no effect on single photons. The experiments were performed using sources that had pure phase fluctuations. We expect that the results presented here will be of interest for applications in the realm of classical and quantum communication and cryptography.

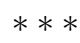

\section{REFERENCES}

[1] Strekalov D. V., Stowe M. C., Chekova M. V. and Dowling J. P., J. Mod. Opt. , 49 (2002) 2349.

[2] Saleh B. E., Abouraddy A. F., Sergienko A. V. and Teich M. C., Phys. Rev. A , 62 (2000) 043816.

[3] Pittmann T., Shin Y., Strekalov D. and A.V. S., Phys. Rev. A, 52 (1995) R3429.

[4] Eisenberg H., Hodelin J., Khoury G. and Bouwmeester D., Phys. Rev. Lett. , 94 (2005) 090502.

[5] Sнін Y., An Introduction to Quantum Optics : Photon and Biphoton Physics (CRC Press (U.S.)) 2011.

[6] Bennick, R. S., Bentley, S. J. and Boyd, R. W., Phys. Rev. Lett. , 89 (2002) 113601.

[7] Abouraddy A., Saleh B., Sergienko A. and Teich M., Phys. Rev. Lett., 87 (2001) 123602.
[8] Saleh B. E. and Teich M. C., Noise in Classical and Quantum Photon-Correlation in Advances in Information Optics and Photonics ed. A.T. Frieberg and R. Dandiker in (SPIE Press, Bellingham, WA) 2008.

[9] Erkmen B. I. and Shapiro J. H., Adv. in Optics and Photonics , 2 (2010) 405.

[10] Klyshko D., Phys. Lett. A , 146 (1990) 93.

[11] Burlakov, A. V., Chekova, M. V., Mamaeva, Yu B., Karabutova, O. A., Korystov, D. Y. and Kulik, S. P., Laser Phys. , 12 (2002) 1.

[12] Shin Y., Rep. Prog. Phys., 66 (2003) 1009.

[13] Scarcelli G., Valencia A. and Shih Y., EPL , 68 (2004) 618.

[14] Valencia A., Scarcelli G., D'Angelo M. and Shih Y., Phys. Rev. Lett., 94 (2005) 063601.

[15] Shirai T., Setala T. and Frieberg, A. T., Phys. Rev. A , 84 (2011) 041801.

[16] Zhou Y., Simon J., Liu J. and Shin Y., Phys. Rev. A, 81 (2010) 043831.

[17] Karmakar S., Zhai Y., Chen H. and Shin Y., The first ghost image using sun as light source in proc. of Quantum Electronics and Laser Science Conference. (Optical Society of America) 2011 p. QFD3.

[18] Venkataraman D., Hardy N., Wong Francis, N. C. and Shapiro, Jeffrey H., Opt. Lett. , 36 (2011) 3684.

[19] Menta P., Samuel J. and Sinha S., Phys. Rev. A , 82 (2010) 034102.

[20] Pancharatnam S., Proc. Ind. Acad. Sci. A, 44 (1956) 247.

[21] Ben-Aryeh Y., Journal of Optics B: Quantum and Semiclassical Optics, 6 (2004) R1.

[22] Samuelsson P., Sukhorukov E. V. and Büttiker M., Phys. Rev. Lett., 92 (2004) 026805.

[23] Aharonov Y. and Bohm D., Phys. Rev. , 115 (1959) 485.

[24] Samuel J., Curr. Sc., 66 (1994) 781.

[25] Batelaan A. and Tonomura A., Phys. Today, 62 (2008) 38.

[26] Wu T. T. and Yang C. N., Phys. Rev. D , 12 (1975) 3845.

[27] Hariharan P., Ramachandran H., Suresh K. and Samuel J., J. Modern Optics , 44 (1997) 707.

[28] Neder I., Ofek N., Chung Y., Heiblum M., Mahalu D. and Umansky V., Nature, 448 (2007) 333.

[29] Pandey D., Satapathy N., Meena M. S. and Ramachandran H., Phys. Rev. A , 84 (2011) 042322.

[30] Loudon R., The Quantum Theory of Light (Oxford University Press) 2010.

[31] Baym G., Acta. Phys. Polonica B, 29 (1998) 1839.

[32] Paul H., Reviews of Modern Physics , 54 (1982) 1061.

[33] Martin A., Alibart O., Flesch J.-C., Samuel J., Sinha S., TAnzilli S. and Kastberg A., EPL (Europhysics Letters), 97 (2012) 10003.

[34] Brendel J., Dultz W. and Martienssen W., Phys. Rev. A , 52 (1995) 2551. 